\title{
BMJ Open Sex differences in the efficacy of antihypertensive treatment in preventing cardiovascular outcomes and reducing blood pressure: protocol for a systematic review and meta-analysis
}

Karina Gasbarrino, ${ }^{1}$ Christopher Labos, ${ }^{2}$ Victoria Mastropietro, ${ }^{3}$ Lindsay Hales, ${ }^{3}$ Nadia Khan, ${ }^{4}$ Doreen Rabi, ${ }^{5}$ Stella S Daskalopoulou (D) ${ }^{1}$

To cite: Gasbarrino K, Labos C, Mastropietro V, et al. Sex differences in the efficacy of antihypertensive treatment in preventing cardiovascular outcomes and reducing blood pressure: protocol for a systematic review and meta-analysis. BMJ Open 2020;10:e036128. doi:10.1136/ bmjopen-2019-036128

- Prepublication history and additional material for this paper are available online. To view these files, please visit the journal online (http://dx.doi. org/10.1136/bmjopen-2019036128).

Received 01 December 2019 Revised 23 January 2020 Accepted 14 February 2020
Check for updates

(C) Author(s) (or their employer(s)) 2020. Re-use permitted under CC BY-NC. No commercial re-use. See rights and permissions. Published by BMJ.

For numbered affiliations see end of article.

Correspondence to Dr Stella S Daskalopoulou; stella.daskalopoulou@mcgill.ca

\section{ABSTRACT}

Introduction Hypertension is a leading cause of mortality worldwide and its prevalence is expected to rise over the next decade. Sex differences exist in the epidemiology and pathophysiology of hypertension. It is well established that antihypertensive treatment can significantly reduce the risk for stroke and other cardiovascular disease events. However, it remains unclear whether this effect is dependent on sex. In this protocol, we outlined a systematic review and meta-analysis to evaluate the effects of antihypertensive therapy in (1) reducing blood pressure and (2) preventing cardiovascular morbidity and mortality outcomes for each sex separately.

Methods and analysis The following electronic databases will be searched: Medline, Embase, The Cochrane Library, PubMed, Cumulative Index of Nursing and Allied Health Literature Plus, Web of Science, grey literature (Google Scholar) and several trial registries. Search strategies will be designed to identify human adult $(\geq 18)$ randomised (and non-randomised) controlled trials, prospective and retrospective cohort studies, and case-control studies concerning 'sex-specific differences associated with the efficacy of antihypertensive treatment'. A preliminary search strategy was developed for Medline (1946 - 16 September 2019). Two investigators will independently review each article included in the final analysis. Primary outcomes investigated are cardiovascular morbidity and mortality and systolic and diastolic blood pressure. Pooled analyses will be conducted using the random-effects model. Publication bias will be assessed by visual inspection of funnel plots and by Begg's and Egger's statistical tests. Between-studies heterogeneity will be measured using the $I^{2}$ test $(p<0.10)$. Sources of heterogeneity will be explored by sensitivity, subgroup and metaregression analyses.

Ethics and dissemination This is the first metaanalysis that will comprehensively compare the efficacy of antihypertensive treatment regimens between men and women. Findings will be shared through scientific conferences and societies, social media and consumer advocacy groups. Results will be used to inform the current guidelines for management of hypertension in men and women by demonstrating the importance of
Strengths and limitations of this study

- The first meta-analysis that will comprehensively compare the efficacy of antihypertensive treatment regimens between men and women.

- There will be no restrictions on the class or dosage of antihypertensive medications used.

- No studies will be excluded based on the risk of bias assessment, and studies will be analysed separately based on study design.

- Sources of heterogeneity will be explored by sensitivity and subgroup analyses.

- An individual patient data meta-analysis will not be undertaken and is a limitation of our study.

implementing sex-specific recommendations. Ethical considerations are not applicable for this protocol.

\section{INTRODUCTION}

Hypertension is a leading cause of mortality worldwide and its prevalence is expected to rise over the next decade in both men and women. ${ }^{12}$ While it is estimated that 1.13 billion people worldwide have hypertension, fewer than one in five people with hypertension are under control. ${ }^{1}$ A strong relationship exists between hypertension and cardiovascular disease (CVD), whereby an increase in blood pressure is associated with an increase in the risk of myocardial infarction, stroke and CVD-related mortality. ${ }^{3} 4$ Specifically, a meta-analysis of individual data from 61 prospective studies reported that at the ages of 40-69 years, a $20 \mathrm{~mm} \mathrm{Hg}$ increase in systolic blood pressure (or equivalently $10 \mathrm{~mm} \mathrm{Hg}$ increase in diastolic blood pressure) was associated with a twofold increase in stroke and ischaemic heart disease death rates. ${ }^{3}$ Therefore, improving hypertension control rates can considerably reduce the 
burden of CVD. A meta-analysis combining data from 123 large-scale blood pressure lowering trials demonstrated that antihypertensive therapy is highly effective in preventing the occurrence of CVD morbidity and mortality; treatment of hypertension was associated with a reduction in the risk of stroke (risk ratio (RR) $0.73 ; 95 \%$ CI 0.68 to 0.77 ), coronary heart disease (RR $0.83 ; 95 \%$ CI 0.78 to 0.88 ), heart failure (RR $0.72 ; 95 \%$ CI 0.67 to 0.78 ) and all-cause mortality (RR $0.87 ; 95 \%$ CI 0.84 to 0.91 ) compared with no drug treatment. ${ }^{5}$

Studies indicate that sex differences exist in the relative contribution of cardiovascular risk factors in women and men. ${ }^{6}$ Furthermore, the prevalence of hypertension differs between men and women across the lifespan. ${ }^{7}$ It is well established that men are more likely to develop hypertension at a younger age compared with premenopausal women. ${ }^{8}$ However, after menopause, women display a more rapid increase in the prevalence of hypertension relative to men, such that after 60-65 years of age hypertension rates in women exceed those observed in men. ${ }^{89}$ As a result, these older women have greater CVD burden than men of similar age. ${ }^{8}$ Despite these sex disparities, hypertension is often poorly controlled in older women. ${ }^{10}$

Although the bioavailability of cardiovascular drugs may differ by sex ${ }^{11}$ there exists no sex-specific guidelines for hypertension management, as it remains unclear whether the effect of antihypertensive treatment in reducing cardiovascular risk is dependent on sex. ${ }^{8}$ Many of the early clinical trials that examined the efficacy of antihypertensive medication in blood pressure control and cardiovascular outcomes collected data in either men alone or combined results for men and women. ${ }^{12}$ Since women were heavily under-represented in these trials, any sex-specific analyses that were performed were severely underpowered. As a result, a systematic review that summarised the results from these early trials concluded that the evidence on the efficacy of antihypertensive treatment specifically in women is weak. ${ }^{13}$ Therefore, gaining better understanding of how women respond to antihypertensive medication is a clinical priority.

Recognising the importance of implementing sexspecific evidence into clinical practice guidelines, in this protocol we outlined a systematic review and metaanalysis to summarise the current evidence evaluating the effects of antihypertensive therapy in (1) reducing blood pressure and (2) preventing cardiovascular morbidity and mortality outcomes for each sex separately. Moreover, we aim to determine whether the treatment effect differs significantly between women and men.

\section{Methods and design}

\section{Registration}

This protocol was written in accordance with the 'Preferred Reporting Items for Systematic Review and Meta-Analysis Protocols' guidelines ${ }^{14}$ (see check-list in online supplementary file appendix 1). Our systematic review and meta-analysis protocol will be registered with the International Prospective Register of Systematic Reviews.

\section{Eligibility criteria}

Studies will be selected according to the criteria outlined below.

\section{Study designs}

We will include randomised controlled trials (RCTs), controlled (non-randomised) clinical trials, prospective and retrospective comparative cohort studies and casecontrol or nested case-control studies performed in humans. Cross-sectional studies, case series, case reports, reviews, commentaries, letters, editorials, conference abstracts and unpublished data will be excluded. All animal and in vitro studies will not be considered.

\section{Participants}

We will include studies involving adult men and women (18 years or older) regardless of race or ethnicity, with clinical indication for antihypertensive therapy. ${ }^{15}$ The studies included will be restricted to a hypertensive cohort who received antihypertensive treatment strictly for hypertension and not for other cardiovascular conditions (other than hypertension) or non-cardiovascular indications (eg, migraine). If the hypertensive cohorts present with other comorbid conditions, this will be allowed to represent clinical reality. However, studies restricted to a specific cohort who is studied after suffering from an acute event (eg, post-myocardial infarction, post-stroke, congestive heart failure) or who is on dialysis will not be considered. Studies that only report sex aggregated data will be excluded.

\section{Interventions}

The intervention will consist of antihypertensive medications, which are used for the pharmacological management of hypertension, as well as the prevention of its complications, such as stroke and myocardial infarction. Several classes of antihypertensive regimens exist including, diuretics, angiotensin-converting enzyme inhibitors, angiotensin II receptor blockers, calcium channel blockers, $\beta$-blockers, as first-line medications, as well as $\alpha$-blockers, $\alpha-2$ receptor agonists, combined $\alpha$-blocker and $\beta$-blocker, central agonists, peripheral adrenergic inhibitors and vasodilators. ${ }^{16}$ There will be no restrictions on the class or dosage of antihypertensive medications used.

\section{Comparators}

Several comparisons will be included:

1. Comparison of the efficacy of antihypertensive therapy in men versus women.

2. Comparison of subjects treated with antihypertensive medication versus placebo (or an active control).

3. Comparisons among different dosages and classes of antihypertensive medications.

4. Subgroup analyses will also be performed as stated below in section 'data synthesis'. 


\section{Outcomes}

Primary: cardiovascular (including cerebrovascular) morbidity and mortality, change in systolic and diastolic blood pressure.

Secondary: all-cause mortality, drug adherence, adverse events.

Studies will be excluded if they do not report any of the primary outcomes.

\section{Timing}

There will be no restrictions by date of publication.

\section{Setting}

There will be no restrictions by type of setting.

\section{Language}

There will be no language restrictions.

\section{Information sources}

The following electronic bibliographic databases will be searched for relevant studies: Medline (via Ovid), Embase Classic+Embase (via Ovid), The Cochrane Central Register of Controlled Trials (via The Cochrane Library), PubMed, Cumulative Index of Nursing and Allied Health Literature Plus (via EBSCO), Web of Science (via Clarivate) and grey literature (Google Scholar). Additionally, the International Clinical Trials Registry Platform Search Portal, the International Standard Randomised Controlled Trial Number Registry and ClinicalTrials.gov will be searched for ongoing or recently completed trials. To ensure literature saturation, reference lists of eligible studies or of relevant meta-analyses and reviews identified through the search will be hand-searched.

\section{Search strategy}

Literature search strategies will be developed using medical subject headings and text words related to 'sex differences', 'antihypertensive medication' and 'blood pressure'. A search strategy will be developed for Medline, and the search terms will be adapted for use with the other bibliographic databases. No date or language limits will be applied on the search. The literature search will be limited to human subjects and adults $\geq 18$ years of age. Conference abstracts, commentaries and letters will be excluded from the search. Search strategies will be peer reviewed by two librarians. A draft Medline search strategy is included in online supplementary file appendix 2.

\section{Study records}

\section{Data management}

Literature search results will be uploaded to Rayyan, an internet-based software program that facilitates collaboration among reviewers during the screening process. Prior to uploading to the software, duplicates will be removed.

\section{Selection process}

First, two authors, KG and CL, will independently screen the titles and abstracts of studies yielded by the search to identify potentially eligible records. They will be unaware of the study's authors' names and journal title to avoid the introduction of a bias in the selection process. Fulltext reports for all studies that appear to meet the inclusion criteria or where there is any uncertainty will then be screened independently by the same authors (KG and CL) to select studies for final inclusion. Disagreements at all stages of the selection process will be resolved through consensus with the corresponding author (SSD). We will contact study authors via email for additional information where necessary to resolve questions concerning the eligibility of the proposed study. If authors do not respond within 4 weeks of initial contact, a follow-up email will be sent. If authors do not respond after 4 weeks of the second contact, the study will not be considered in the analysis.

\section{Data collection process}

Data extraction will be performed independently by $\mathrm{KG}$ and CL. Data will be preferentially extracted from result tables in the selected articles. If the data are not listed in the tables, the text in the results section will be carefully read for any important information. If the data are only available from graphs, the data will be extracted manually using the Image J V.1.47 t (ImageJ, US National Institutes of Health, Bethesda, Maryland, http://imagej.nih. gov/ij/, 1997-2015). Extracted data will include study and population characteristics, details on blood pressure, intervention details and patient outcomes of interest. Authors will be contacted in case further information and clarifications are needed using the same strategy as mentioned previously.

\section{Data items}

Data items that will be extracted from each included article are presented in table 1 .

\section{Outcomes and prioritisation}

To be included, study outcomes have to be available according to sex. For primary outcomes, data involving the change in systolic and diastolic blood pressure (in $\mathrm{mm} \mathrm{Hg}$ ) between baseline and follow-up will be extracted, as well as effect estimates for cardiovascular (and cerebrovascular) morbidity and mortality, including fatal and/or non-fatal myocardial infarction, fatal and/ or non-fatal stroke, fatal and/or non-fatal heart failure (including hospitalisations), major adverse cardiac events and cardiovascular death. Effect estimates for all-cause mortality will be extracted as a secondary outcome, along with drug compliance (in \%) and safety outcomes, that is, the incidence of adverse events (including but not limited to, allergic reaction/ angioedema/skin rash, electrolyte disturbances, cough, peripheral oedema, diarrhoea or constipation, nausea or vomiting, skin rash, agitation or anxiety, insomnia, palpitations) and permanent treatment discontinuations because of adverse events (in \%).

Risk of bias in individual studies

Risk of bias assessment for randomised controlled trials will be performed using the modified Cochrane Risk of Bias tool. Judgements, expressed as high, low or unclear 
Table 1 Data items to be extracted from each included article

\begin{tabular}{ll}
\hline Data item & Details to be extracted \\
\hline Study characteristics & $\begin{array}{l}\text { Complete author list, publication year, journal, funding source, geographical location of study, study } \\
\text { design, randomisation method if applicable, total number of subjects per group }\end{array}$ \\
Patient characteristics & $\begin{array}{l}\text { Average age, sex distribution, average body mass index, type of hypertension (eg, sustained } \\
\text { hypertension, masked hypertension, white-coat hypertension, nocturnal hypertension), duration of } \\
\text { hypertension, average systolic and diastolic blood pressure at baseline, list of other comorbidities }\end{array}$ \\
Intervention & $\begin{array}{l}\text { Generic name of antihypertensive medication, type of control used, dosage, frequency and duration of } \\
\text { treatment, delivery method }\end{array}$ \\
Results & $\begin{array}{l}\text { Duration of follow-up, achieved systolic and diastolic blood pressure, cardiovascular (and } \\
\text { cerebrovascular) morbidity and mortality, all-cause mortality, drug compliance, adverse events, } \\
\text { permanent treatment discontinuations }\end{array}$
\end{tabular}

risk, will be made independently by two authors, $\mathrm{KG}$ and CL, based on the criteria for judging the risk of bias. Disagreements will be resolved first by discussion and then by consulting a third author (SSD) for arbitration. For cohort and case-control studies, risk of bias assessment will be performed using the 9-item NewcastleOttawa Quality Assessment Scale. Three parameters will be evaluated: (1) population selection, (2) comparability of results and (3) ascertainment of exposure or outcome. Similarly, two independent reviewers, KG and CL, will perform each quality assessment, consulting a third reviewer (SSD) when necessary. Studies will be considered of high quality if the total score is $\geq 7 / 9$. No studies will be excluded based on the risk of bias assessment.

\section{Data analysis}

Synthesis

Meta-analyses will be conducted separately for men and women to evaluate the effect of antihypertensive therapy on (1) blood pressure and (2) cardiovascular morbidity and mortality outcomes. Data concerning systolic and diastolic blood pressure will be expressed as mean differences (with 95\% CIs). Data concerning each cardiovascular morbidity and mortality outcome will be expressed as summary RR with $95 \%$ CIs. Numbers needed to treat estimates, which is the number of patients who must be treated to prevent one adverse outcome, will be calculated. Between-studies heterogeneity will be measured using the $\mathrm{I}^{2}$ test; significance threshold will be set at $\mathrm{p}<0.10$. Pooled analyses will be conducted using the random effects (DerSimonian-Laird) model irrespective of the underlying heterogeneity across studies. Standardisation of treatment effect according to blood pressure lowering will not be performed. ${ }^{1718}$

Evidence obtained from randomised interventional studies will not be evaluated together with nonrandomised and/or epidemiological evidence as this will introduce several types of bias in our analyses. Thus, analyses will be stratified based on study design and risk of bias with randomised controlled trials being included in the primary synthesis. Other sources of heterogeneity will also be explored by sensitivity and subgroup analyses, stratifying studies by various factors, including follow-up duration, age (eg, younger vs older women, younger vs older men, younger women vs younger men, older women vs older men), ethnicity/race, type of hypertension (eg, sustained hypertension, masked hypertension, white-coat hypertension, nocturnal hypertension), class of antihypertensive therapy and treatment dosage. Metaregression analyses will also be performed to assess the effect of confounding variables (ie, body mass index, smoking status, cholesterol levels, history of prior CVD, type 2 diabetes mellitus, chronic kidney disease).

All statistical analyses for meta-analysis will be performed using STATA Software V.13.0 (STATA Corporation, College Station, Texas, USA).

\section{Meta-bias(es)}

Publication bias will be assessed by visual inspection of funnel plots and by Begg's and Egger's statistical tests, if at least nine studies are available. $\mathrm{P}<0.05$ will be considered evidence of small study effects. To determine whether outcome reporting bias is present, we will evaluate whether the protocol of the RCT was published before recruitment of patients of the study was started. Moreover, we will compare outcomes reported in the protocol and the published report.

\section{Confidence in cumulative estimate}

The quality of evidence for all outcomes will be judged using the Grading of Recommendations Assessment, Development and Evaluation Working Group criteria. ${ }^{19}$

\section{Patient and public involvement}

Patients or the public were not involved in the design, or conduct, or reporting, or dissemination plans of our research.

\section{DISCUSSION}

Hypertension is a major risk factor for CVD, placing it as the most common cause of death worldwide. ${ }^{2}$ Overwhelming evidence indicates that sex differences exist in the epidemiology and the pathophysiology of hypertension. ${ }^{20}$ Specifically, premenopausal women have lower incidence and severity of hypertension, and thus a lower 
incidence of CVD, than age-matched men. ${ }^{8}$ However, this cardioprotection is compromised post-menopause, where the risk of hypertension increases sharply in women. ${ }^{89}$ We expect that this sexual dimorphism may also extend to the treatment efficacy of antihypertensive medication. It is well established that antihypertensive treatment can significantly reduce the risk for stroke and other CVD events. ${ }^{5}$ However, it remains unclear whether this effect is dependent on sex. Moreover, it is not established whether different classes of antihypertensive drugs routinely used in clinical practice work similarly in men and women. Currently, hypertension guidelines do not report sex-specific recommendations. Evaluation of these differences is required to ensure the best possible care for both men and women living with hypertension. Thus, herein, we provide a protocol of a systematic review and meta-analysis that summarises the current evidence evaluating sex differences in the efficacy of antihypertensive treatment in reducing CVD outcomes and blood pressure. To our knowledge, this is the first meta-analysis that will comprehensively compare the efficacy of antihypertensive treatment regimens between men and women. In addition to analysing more recent trial data on this topic than a previously performed meta-analysis of randomised trials (prior to 2006), ${ }^{21}$ we will also include non-randomised and epidemiological evidence, and we will perform several subanalyses, as appropriate.

\section{Ethics and dissemination}

We will share the findings of these meta-analyses through scientific conferences and societies, social media and consumer advocacy groups. Importantly, the results will be used to inform the current guidelines for management of hypertension in men and women by demonstrating the importance of implementing sex-specific recommendations. It may determine which classes of antihypertensive medications may be more effective in men and in women. Treatment strategies for hypertension and CVD that are tailored according to sex could lead to improved outcomes for the affected individuals. Ethical considerations are not applicable for this protocol.

\section{Author affiliations \\ ${ }^{1}$ Medicine, Research Institute of the McGill University Health Centre, Montreal, \\ Canada \\ ${ }^{2}$ Cardiology, Queen Elizabeth Health Complex, Montreal, Canada \\ ${ }^{3}$ Medical Library, McGill University Health Centre, Montreal, Canada \\ ${ }^{4}$ Medicine, The University of British Columbia, Vancouver, Canada \\ ${ }^{5}$ Medicine, University of Calgary Cumming School of Medicine, Calgary, Canada}

Contributors SSD is the guarantor. KG and SSD designed the research. KG drafted the manuscript. KG, CL and SSD contributed to the development of the inclusion/ exclusion search criteria, data extraction criteria and statistical analysis methods. VM and LH led the development of the search strategy. NK and DR critically revised the protocol. All authors read, provided feedback and approved the final protocol.

Funding This protocol is supported by Hypertension Canada. SS Daskalopoulou is a Senior Chercheur-Boursier Clinicien supported by the Fonds de recherche du Québec-Santé. NK is funded by a Michael Smith Foundation in Health Research Career Scientist Award.

Competing interests None declared.
Patient consent for publication Not required.

Provenance and peer review Not commissioned; externally peer reviewed.

Open access This is an open access article distributed in accordance with the Creative Commons Attribution Non Commercial (CC BY-NC 4.0) license, which permits others to distribute, remix, adapt, build upon this work non-commercially, and license their derivative works on different terms, provided the original work is properly cited, appropriate credit is given, any changes made indicated, and the use is non-commercial. See: http://creativecommons.org/licenses/by-nc/4.0/.

ORCID iD

Stella S Daskalopoulou http://orcid.org/0000-0003-4774-2549

\section{REFERENCES}

1 World Health Organization. Hypertension, 2019. Available: https:// www.who.int/news-room/fact-sheets/detail/hypertension

2 Ezzati M, Lopez AD, Rodgers A, et al. Selected major risk factors and global and regional burden of disease. Lancet 2002;360:1347-60.

3 Lewington S, Clarke R, Qizilbash N, et al. Age-Specific relevance of usual blood pressure to vascular mortality: a meta-analysis of individual data for one million adults in 61 prospective studies. Lancet 2002;360:1903-13.

4 MacMahon S, Peto R, Cutler J, et al. Blood pressure, stroke, and coronary heart disease. Part 1, prolonged differences in blood pressure: prospective observational studies corrected for the regression dilution bias. Lancet 1990;335:765-74.

5 Ettehad D, Emdin CA, Kiran A, et al. Blood pressure lowering for prevention of cardiovascular disease and death: a systematic review and meta-analysis. Lancet 2016;387:957-67.

6 Vitale C, Mendelsohn ME, Rosano GMC. Gender differences in the cardiovascular effect of sex hormones. Nat Rev Cardiol 2009;6:532-42.

7 Mozaffarian D, Benjamin EJ, Go AS, et al. Heart disease and stroke statistics-2016 update: a report from the American heart association. Circulation 2016;133:e38-60.

8 Beth Abramson L L, Davis LL, Parapid B. Women and hypertension: beyond the 2017 guideline for prevention, detection, evaluation, and management of high blood pressure in adults, 2018.

9 Wenger NK, Arnold A, Bairey Merz CN, et al. Hypertension Across a Woman's Life Cycle. J Am Coll Cardiol 2018;71:1797-813.

10 Lloyd-Jones DM, Evans JC, Levy D. Hypertension in adults across the age spectrum: current outcomes and control in the community. JAMA 2005;294:466-72.

11 Luzier AB, Killian A, Wilton JH, et al. Gender-Related effects on metoprolol pharmacokinetics and pharmacodynamics in healthy volunteers. Clin Pharmacol Ther 1999;66:594-601.

12 Garcia M, Mulvagh SL, Merz CNB, et al. Cardiovascular disease in women: clinical perspectives. Circ Res 2016;118:1273-93.

13 Ljungman C, Mortensen L, Kahan T, et al. Treatment of mild to moderate hypertension by gender perspective: a systematic review. $J$ Womens Health 2009;18:1049-62.

14 Shamseer L, Moher D, Clarke M, et al. Preferred reporting items for systematic review and meta-analysis protocols (PRISMA-P) 2015: elaboration and explanation. BMJ 2015;350:97647.

15 Nerenberg KA, Zarnke KB, Leung AA, et al. Hypertension Canada's 2018 guidelines for diagnosis, risk assessment, prevention, and treatment of hypertension in adults and children. Can J Cardiol 2018;34:506-25.

16 American Heart Association. Types of blood pressure medications, 2017. Available: https://www.heart.org/en/health-topics/high-bloodpressure/changes-you-can-make-to-manage-high-blood-pressure/ types-of-blood-pressure-medications

17 Brunström M, Carlberg B. Standardization according to blood pressure lowering in meta-analyses of antihypertensive trials: comparison of three methodological approaches. J Hypertens 2018;36:4-15.

18 Thomopoulos C, Michalopoulou H. Outcome standardization to blood pressure reduction in meta-analyses: sailing in uncharted waters. J Hypertens 2018;36:31-3.

19 Grade home. Available: http://www.gradeworkinggroup.org

20 Regitz-Zagrosek V, Kararigas G. Mechanistic pathways of sex differences in cardiovascular disease. Physiol Rev 2017;97:1-37.

21 Turnbull F, Woodward M, Neal B, et al. Do men and women respond differently to blood pressure-lowering treatment? results of prospectively designed overviews of randomized trials. Eur Heart $J$ 2008:29:2669-80. 